OPEN ACCESS

Edited by:

Giovanni Albani,

Istituto Auxologico Italiano

(IRCCS), Italy

Reviewed by:

Ana María Espinosa Oliva,

University of Seville, Spain

Jong-Min Kim,

Seoul National University

Bundang Hospital, South Korea

*Correspondence:

Heather E. Rasmussen

heather.rasmussen@unl.edu

Specialty section: This article was submitted to Movement Disorders, a section of the journal

Frontiers in Neurology

Received: 12 April 2019

Accepted: 06 June 2019

Published: 20 June 2019

Citation:

Cantu-Jungles TM, Rasmussen HE and Hamaker BR (2019) Potential of

Prebiotic Butyrogenic Fibers in

Parkinson's Disease.

Front. Neurol. 10:663.

doi: 10.3389/fneur.2019.00663

\section{Potential of Prebiotic Butyrogenic Fibers in Parkinson's Disease}

\author{
Thaisa M. Cantu-Jungles ${ }^{1}$, Heather E. Rasmussen ${ }^{2 \star}$ and Bruce R. Hamaker ${ }^{1}$ \\ ${ }^{1}$ Department of Food Science, Whistler Center for Carbohydrate Research, Purdue University, West Lafayette, IN, \\ United States, ${ }^{2}$ Department of Nutrition and Health Sciences, University of Nebraska-Lincoln, Lincoln, NE, United States
}

Parkinson's Disease (PD) is a neurodegenerative disorder characterized by loss of dopaminergic neurons in the substantia nigra. Recent evidence supports the involvement of the gastrointestinal tract in PD pathogenesis, including alterations in microbiota and intestinal permeability. Apart from being the preferred energy source for colonic epithelial cells, butyrate is involved in anti-inflammatory, enteroendocrine and epigenetic mechanisms that influence colonic and systemic health, including brain function. A few studies using oral administration of sodium butyrate indicate beneficial effects in PD animal models; however, prebiotic fibers that generate butyrate locally in the gut may be more effective. The design and selection of butyrogenic prebiotic fibers would allow preclinical studies to evaluate how gut-derived butyrate could affect PD pathophysiology. This review describes potential benefits of increasing gut butyrate production in PD through a prebiotic approach. Moreover, physico-chemical features of prebiotic fibers that target butyrogenic colonic bacteria are discussed.

Keywords: dietary fiber, Parkinson's disease, butyrate, gut microbiota, prebiotics

\section{INTRODUCTION}

Parkinson's disease (PD) is a relentlessly progressive neurodegenerative disease of aging, with a considerable burden of disability. It is believed that PD pathology is a consequence of both genetic susceptibility and toxic environmental factors, resulting in increasing neuronal oxidative stress (1). The pathological hallmark of PD is neuronal inclusions termed Lewy bodies (LB) or Lewy neurites (LN) whose main component is aggregated and phosphorylated $\alpha$-synuclein and is responsible for neurological symptoms and signs of PD (2).

Gastrointestinal involvement in PD may be pathogenic or a consequence of the disease. More recently, researchers have provided evidence that supports a role for the gastrointestinal tract and the enteric nervous system (ENS) in the pathogenesis of PD $(3,4) . \alpha$-Synuclein aggregates are present in Substance P containing neurons in the sigmoid colonic submucosal neurons in patients with PD (5). Microbiota differs between those with PD and healthy controls; for instance, those with PD have a lower abundance of Clostridium cluster XIVa and IV (6-10). Changes in caecum mucosal-associated and luminal microbiota, including a significant decrease in the relative abundance of the beneficial commensal bacteria genus Bifidobacterium, has been induced by a mouse model of PD (11). Recently, evidence for proinflammatory dysbiosis in PD patients has been shown, and researchers suggest that this dysbiosis could trigger inflammation-induced misfolding of $\alpha$-Syn and development of PD pathology (6). Additionally, intestinal permeability was increased and beneficial metabolites of microbiota function, such as short chain fatty acids (SCFA), were lower in those with PD compared to healthy controls (5). As evidence 
for gastrointestinal tract involvement in PD exists, this suggests that therapeutic interventions may be warranted that positively impact the intestinal milieu by changing microbiota to produce less pro-inflammatory/injurious products and/or prevent gut leakiness.

\section{PREBIOTIC FIBER: DEFINITION, STRUCTURE AND FUNCTION}

The term prebiotics was first introduced in 1995 by Gibson and Roberfroid as "a non-digestible food ingredient that beneficially affects the host by selectively stimulating the growth and/or activity of one or a limited number of bacteria in the colon, and thus improves host health" (12). Since then, the original definition has been revised several times and recently broadened to 'a substrate that is selectively utilized by host microorganisms conferring a health benefit' (13). This should not be confused with probiotics, defined as "live microorganisms that confer a health benefit on the host when administered in adequate amounts" (14).

Although prebiotic definitions are general to all oligoand polysaccharide prebiotic substrates, researchers up to 2010 have largely focused only on the use of fructans (fructooligosaccharides [FOS] and inulin), galactooligosaccharides (GOS) and, to a minor extent, lactulose, to promote beneficial shifts in the gut bacterial community (15). Prebiotic oligosaccharides were mainly used to promote increases in Lactobacillus and Bifidobacterium species (16). More recently, however, as the complexity and function of gut microbial ecosystems have been unveiled, new microbial groups or species of health interest have been identified, as well as ways to promote them (17-19). The challenge of achieving prebiotic effects favoring specific microbial groups requires the understanding of how prebiotic structure relates to substrate requirements of target bacteria and how they compete on substrates relative to other microbial groups (20).

The majority of prebiotic substrates fall into the dietary fiber classification-i.e., carbohydrate polymers not hydrolyzed by endogenous enzymes in the small intestine (21). Carbohydrates are the most abundant and heterogeneous class of molecules found in nature. In plants, non-cellulosic carbohydrate fibers include $\beta$-glucans, fructans, mannans, xylans, galactans, arabinans, arabinogalactans, pectins, and resistant starch. Also, carbohydrate fibers such as agars, sulfated carbohydrates, alginates, fucoidans, $\alpha, \beta$-glucans and chitin may be found in other natural sources $(22,23)$. Apart from being a highly diverse class of molecules, complex variations at the fine chemical structure level (e.g., polymer size, linkage type, composition and arrangement of side chains, degree, and identity of esterlinked molecules) are possible within polymer class, resulting in dietary fibers with distinct solubilization degree, viscosity and tridimensional structure (20). For the complete hydrolysis and utilization of such complex molecules, a given gut bacteria should have within its genome the ability to produce recognition and binding proteins, transporters and carbohydrate-active enzymes (CAZymes) specific to a particular physicochemical structure (24). As such, the ability and efficiency in utilizing carbohydrates widely varies within gut individual bacteria or bacterial groups $(24,25)$. In addition, overlapping abilities in fiber degradation within bacterial species result in competitive pressures within the gut. For instance, Xu et al. (26) showed that strains of $B$. cellulosilyticus and $B$. ovatus both had the ability to grow on simple arabinoxylan structures. However, when the strains were cultivated together, $B$. ovatus outcompeted and dominated over $B$. cellulosilyticus. Thus, prebiotic fibers with specific physicochemical features can be selected to promote certain bacteria based on the ability of a bacteria or bacterial group to access and utilize them efficiently in the competitive environment of the colon (20).

\section{METABOLITES FROM COLONIC DIETARY FIBER FERMENTATION IN PARKINSON'S DISEASE}

The colonic fermentation of dietary fiber by specialist microbes in the gut leads to the formation of a variety of gases and metabolites. SCFAs including acetate, propionate, and butyrate comprise $90-95 \%$ of all microbiota metabolites produced in the colon (27-29). SCFAs hold biological significance and may act both locally in the gut and systemically to promote health benefits at distinct body sites. In neurological disorders, SCFAs are potentially important for their role in anti-inflammatory processes (30-32), promotion of blood-tissue barrier integrity $(33,34)$, and neuromodulation $(35,36)$. Moreover, local effects such as triggering gut peristaltic reflexes (37) could be relevant, as constipation is an usual clinical finding in many neurological disorders, including PD $(38,39)$. Although there are no studies evaluating acetate and propionate singly in PD, butyrate has been studied and the majority of preclinical evidence suggests that it specifically could be beneficial in many aspects of PD (40-45).

\section{Butyrate}

Butyrate is the preferred energy source for gut enterocytes, responsible for most of their energy metabolism (46). Butyrate also supports gut barrier function through the stimulation of tight junction assemblies and mucus production. As mentioned, hyperpermeability of the colonic epithelium occurs in PD (5); thus, the action of butyrate on the gut barrier may have clinical importance in PD. At the cell surface level, butyrate elicits a variety of physiological responses through $G$ proteincoupled receptors (GPCR) in enterocytes (47). In particular, butyrate regulates inflammatory pathways that are important in maintaining gut homeostasis $(48,49)$ and stimulates the production of enteroendocrine hormones such as glucagon-like peptide 1 (GLP-1) and peptide YY $(50,51)$ (Figure 1). Both of these hormones reach circulation and exert their action through receptors spread at distinct body sites, including the brain. In a mouse model of PD, oral administration of sodium butyrate 


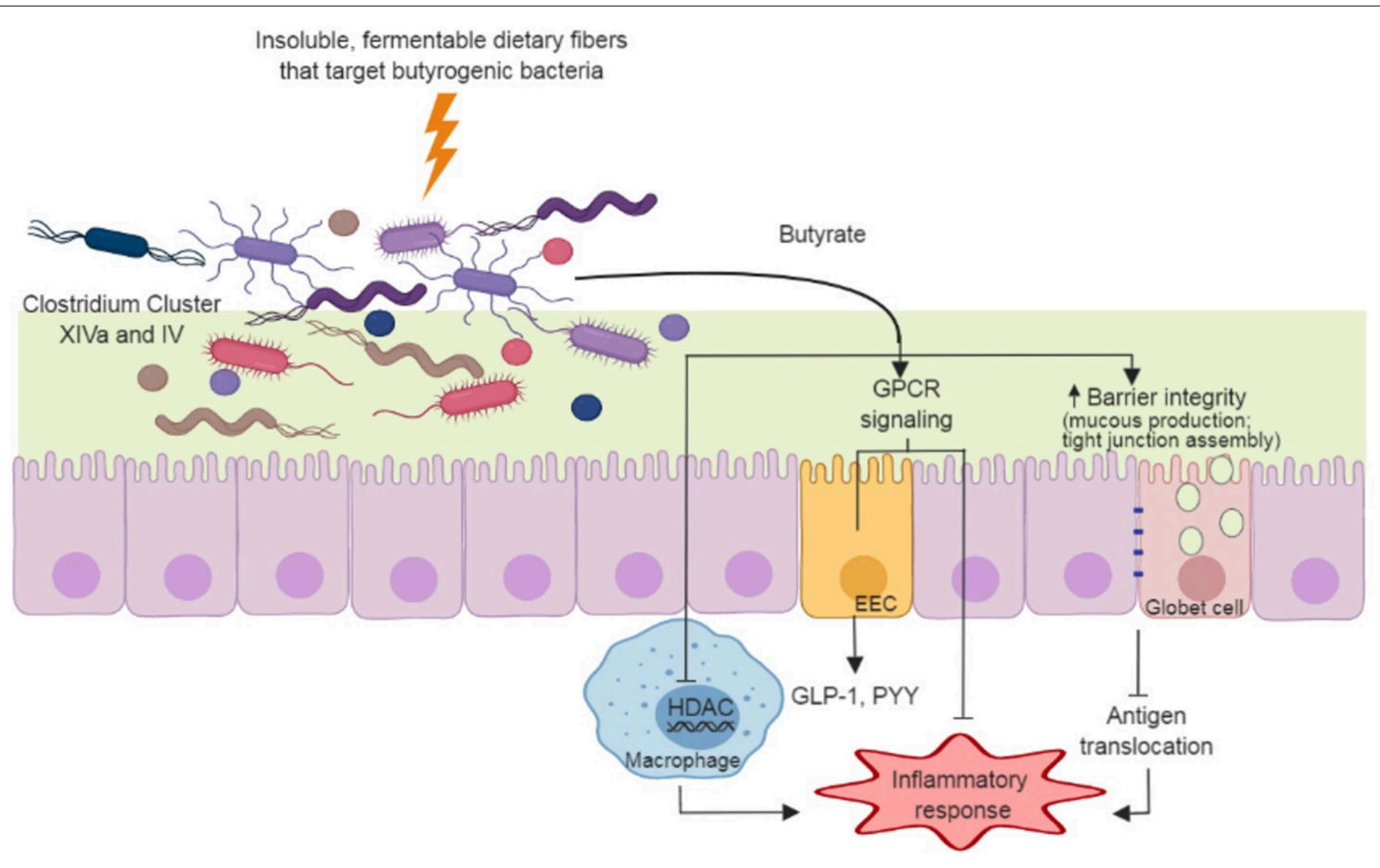

FIGURE 1 | Dietary fiber approach to increase gut-produced butyrate and pathways that have potential benefits to Parkinson's Disease. Insoluble dietary fibers with specific chemical structures are fermented by butyrate producers in the gut (e.g., Clostridium Cluster XIVa and IV species). The butyrate produced during fermentation supports gut barrier function through the stimulation of mucus production and tight junction assemblies, and minimizing antigen translocation and inflammation. Butyrate also regulates inflammatory pathways through G protein-coupled receptors (GPCR) in enterocytes and through inhibition of histone deacetylase (HDAC) in macrophages. GPCR signaling in enteroendocrine cells (EEC) induce secretion of hormones (e.g., glucagon-like peptide-1 [GLP-1] and peptide YY[PYY]) that act in many organs, including the brain.

increased colonic GLP-1 levels as well as upregulated GLP1 receptors (GLP-1R) in the brain and resulted in improved neurobehavioral impairment (52).

Butyrate also influences histone acetylation, a posttranslational modification that influences the propensity of a gene to be transcribed or repressed (53). Butyrate acts as a histone deacetylase inhibitor (HDACi) (54), attenuating production and secretion of pro-inflammatory cytokines in response to lipopolysaccharide stimuli in macrophages, complementing analogous modulation of inflammatory process via GPCRs (55) (Figure 1). Butyrate-targeted histone deacetylase inhibition is also neuroprotective against dopamine cell death (44) and DNA damage (42) in-vitro. In a rotenone-induced drosophila model of PD, sodium butyrate was able to improve locomotor deficits and reduce early mortality (40). Similar results were observed in a 6-hydroxydopamine-induced rat model of PD, in which sodium butyrate attenuated motor impairment and increased dopamine levels (45). In addition, Zhou et al. (43) showed that in a cell culture and a murine model of PD, sodium butyrate was able to up-regulate gene expression of DJ-1, a protein known to protect dopamine neurons from oxidative stress and moderate protein aggregation.

All animal studies using PD models utilized oral administration of sodium butyrate, rather than an approach using butyrogenic prebiotics. It should be noted that sodium butyrate is delivered differently to the body compared to microbiota-produced butyrate from prebiotic fermentation. Sodium butyrate is absorbed mostly in upper segments of the gastrointestinal tract, it leads to significant increases in plasma concentrations of butyrate (56). While this could result in direct actions in the brain, upper gastrointestinal tract absorption prevents most of the butyrate supplemented to reach the large intestine, where it has functions that could be relevant in PD (e.g., gut barrier function, regulation of inflammatory pathways, enteroendocrine hormone release). Microbiota-derived butyrate, on the other hand, generally is considered to act locally in the gut, with the remaining portion absorbed by the liver with no significant amounts reaching bloodstream (57). Interestingly, some reports show increased blood levels of circulating butyrate in healthy subjects in response to dietary fiber interventions (58-60), indicating that a portion of butyrate may escape liver absorption and could have a direct action in the brain. Inflammatory conditions may also cause an increase of SCFAs in peripheral venous blood (61), and therefore, the extent of microbiota produced-butyrate that reaches bloodstream in $\mathrm{PD}$ patients is still a matter of investigation. Overall, the use of prebiotic dietary fibers to increase butyrate in the colon could promote both localized and systemic effects (Figure 1), which seems like a promising approach in the management of PD. However, preclinical 
studies are needed to evaluate how gut-derived butyrate affects PD pathophysiology.

Some controversy regarding the commonly accepted concept of anti-inflammatory and neuroprotective action of SCFAs was brought to light in a study using a mouse model of PD $(62,63)$. Sampson et al. (62) reported that the oral administration of a SCFA mixture, as well as a fecal transplant, to animals raised in a germ-free environment or antibiotic-treated, enhanced PD pathophysiology. It was not clear, however, if the SCFA mixture dosage utilized corresponds to levels that can be reached through gut-microbiota production. In this regard, oral administration of $100 \mathrm{mg} / \mathrm{kg}$ of sodium butyrate $(\mathrm{NaB})$, but not $1,200 \mathrm{mg} / \mathrm{kg}$, attenuated social deficits in an autism mouse model (64), indicating that distinct outcomes may take place by changing SCFA concentration. Another consideration is that orally delivered butyrate is mainly absorbed in the upper gastrointestinal tract and could have distinctly different outcomes from the colonic-produced butyrate.

\section{BUTYROGENIC BACTERIA IN THE LARGE INTESTINE}

A number of commensal gram-positive bacteria in the human gut possess the ability to produce butyrate. The majority of the butyrate producing bacteria belong to Clostridium Clusters IV and XIVa of the Firmicutes phylum. These clusters comprise highly oxygen-sensitive bacteria, which are estimated to significantly contribute to colonic butyrate production $(65,66)$. They also correspond to a numerically important portion of colonic bacteria. Faecalibacterium prausnitzii from Clostridium Cluster IV and Eubacterium rectale from Clostridium Cluster XIVa comprise up to 14 and 13\%, respectively, of total fecal gut microbiota (67). Other major butyrogenic bacteria isolated from the human colon are Roseburia spp., Eubacterium spp., Anaerostipes caccae, Butyrivibrio fibrisolvens, Coprococcus spp. from Clostridium Cluster XIVa and Subdoligranulum variabile and Anaerotruncus colihominis from Clostridium Cluster IV (66). Many of commensal clostridial species preferentially colonize the mucus layer (e.g., E. rectale, F. prausnitzii, and R. intestinalis) which is in close proximity to gut epithelium. This strategic position favors butyrate interaction and uptake by intestinal cells, stimulating physiologic, metabolic and immunologic processes of health significance. Nonetheless, species such as A. caccae mostly inhabit the lumen of the colon where butyrate production helps to reduce luminal $\mathrm{pH}$, preventing the growth pathogenic bacteria $(68-70)$. Non-butyrogenic species also indirectly contribute to butyrate formation through production of other SCFA as a more acidic gut milieu favors the growth of butyrogenic species (7173). Also, many butyrogenic bacteria utilize lactate and acetate from other bacteria to produce butyrate (66). The importance of such crossfeeding mechanisms to improve butyrate formation in the gut is still a matter of discussion as many butyrogenic bacteria occupy spatially distinct niches different than non-butyrogenic ones within the gut $(70,74,75)$.

Depletion of butyrogenic bacteria from Clostridium Cluster IV and XIVa, especially those found nearly associated to the mucus layer is a common and potentially negative finding in the elderly (68). On top of that, PD patients show lower abundance of Lachnospiraceae family members (Clostridium Cluster XIVa) (6-8) and Faecalibacterium (Clostridium Cluster IV) (6, 8-10), as well as low production of all three SCFAs, including butyrate (9) compared to individuals of similar age.

\section{PREBIOTIC DIETARY FIBER TARGETING BUTYROGENIC BACTERIA AND BUTYRATE PRODUCTION}

Colonic bacteria produce butyrate mainly through dietary fiber fermentation, with proteolytic pathways contributing very little to overall butyrate production (65). Consumption of a meatbased diet for five consecutive days resulted in lower butyrate levels in fecal samples of healthy volunteers when compared to a plant-based diet. Butyrate reduction was accompanied by decrease in abundance of butyrogenic bacteria from Firmicutes, such as Roseburia and E. rectale (Clostridium Cluster XIVa). Another study with obese individuals showed that 4 weeks of a very low total carbohydrate intake ( $24 \mathrm{~g} /$ day), including low dietary fiber, resulted in a 4 -fold decrease in Roseburia

TABLE 1 | Examples of insoluble substrates capable of promoting butyrogenic colonic bacteria.

\begin{tabular}{|c|c|c|c|}
\hline Dietary fiber & Study design & Butyrogenic bacteria positively affected & Study \\
\hline Chitin-glucan complexes & Fecal analysis from diet-induced obese mice & $\begin{array}{l}\text { Clostridium Cluster XIVa, including Roseburia } \\
\text { spp. }\end{array}$ & Neyrinck et al. (80) \\
\hline$\beta-1,3 / 1,6-D-g l u c a n$ & In vitro human fecal fermentation & Anaerostipes spp. and Roseburia & Cantu-Jungles et al. (81) \\
\hline Whole grain barley & Fecal analysis from healthy human subjects & $\begin{array}{l}\text { Eubacterium rectale, Roseburia faecis and } \\
\text { Roseburia intestinalis }\end{array}$ & Martinez et al. (82) \\
\hline Wheat bran & Fecal analysis from obese males & Members from Lachnospiraceae family & Salonen et al. (83) \\
\hline $\begin{array}{l}\text { Acetylated galactoglucomannan and highly } \\
\text { acetylated arabinoglucuronoxylan (AGX) }\end{array}$ & In vitro human fecal fermentation & Faecalibacterium prausnitzii & La Rosa et al. (84) \\
\hline Wheat bran & In vitro human fecal fermentation & $\begin{array}{l}\text { Members from Lachnospiraceae family and } \\
\text { uncultured butyrate producers }\end{array}$ & Duncan et al. (85) \\
\hline Coarse wheat bran & In vitro human fecal fermentation & $\begin{array}{l}\text { Coprococcus eutactus, Roseburia and other } \\
\text { Lachnospiraceae family members }\end{array}$ & Tuncil et al. (86) \\
\hline
\end{tabular}


spp. and E. rectale accompanied by the same magnitude reduction in butyrate fecal content (76). These data suggest that these colonic bacteria are particularly dependent upon dietary fiber consumption.

Contrary to what is found in Bacteroidetes (known as carbohydrate generalists, as many species have overlapping nutrient utilization abilities), available data suggest that, in addition to crossfeeding, butyrogenic bacteria are more specialized to degrade unique fiber structures. For example, Sheridan et al. (77) showed that even bacteria from the same Roseburia genus (Clostridium Cluster XIVa) present variable abilities to grow in distinct substrates in single cultures, with little overlapping in fiber utilization capabilities within species.

As previously discussed, fiber physical features are also related to its fermentation profile. Most bacteria attached to particles recovered from human feces belong to Firmicutes (mean $76.8 \%$ against only $18.5 \%$ Bacteroidetes), with high abundance of species from Clostridium Cluster IV and XIVa (74). In vitro fecal fermentation of wheat bran also showed that Clostridium Cluster XIVa dominated amongst particle-associated bacteria (78). As primary colonizers of insoluble substrates, these bacteria would hold a competitive advantage to degrade insoluble fermentable substrates. In fact, in pure cultures of $R$. intestinalis and Bacteroides xylanisolvens, the former was shown to be strongly associated with insoluble xylan, while B. xylanisolvens was enriched in solubilized xylan fractions (79).

Corroborating these results, many insoluble substrates such as chitin-glucan and $\beta$-glucan, as well as some cereals rich in insoluble fractions, were shown to increase butyrate and/or colonic butyrogenic bacteria (Table 1). Chitin-glucan complexes were shown to specifically increase Clostridium Cluster XIVa, including Roseburia spp. in high-fat (HF) diet-induced obese mice and promoted desirable metabolic outcomes (80). In our research group, insoluble $\beta$-glucans from fungi specifically increased Anaerostipes spp. (Clostridium Cluster XIVa) from $<0.5 \%$ of the total bacteria in the initial inoculum to approximately $24 \%$ after fermentation of such fiber in vitro (81). This was accompanied by butyrate increase from 12.5 to $24-26 \%$ after $\beta$-glucan fermentation (81). Whole grain barley (82) and wheat bran $(83,85)$ were shown to be fermented by members of Lachnospiraceae family (Clostridium Cluster XIVa) in human colonic microbiota. Lignocellulosic dietary fibers from feedstocks such as galactoglucomannan and arabinoglucuronoxylan were shown to increase Faecalibacterium prausnitzii (Clostridium Cluster IV) (84). In an indirect way, acetate producers, such as Ruminococcus bromii through utilization of resistant starch, can promote butyrate production through cross-feeding (87). These studies confirm that insoluble polymers with distinct chemical structures boost divergent butyrogenic bacteria in the colon.

\section{REFERENCES}

1. Thomas B, Beal M. Parkinson's disease. Hum Mol Genet. (2007) 16:R138-94. doi: $10.1093 / \mathrm{hmg} / \mathrm{ddm} 159$

2. Braak H, Del Tredici K. Nervous system pathology in sporadic Parkinson disease. Neurology. (2008) 70:1916-25. doi: 10.1212/01.wnl. $0000312279.49272 .9 f$
Finally, besides solubility degree and chemical structure, particle size may be an important fiber characteristic to consider in butyrogenic prebiotic fiber design and selection. Tuncil et al. (86) showed that in vitro fecal fermentation of larger wheat bran particle size fractions led to higher butyrate production, as well as increases in some members of the Lachnospiraceae family (Clostridium Cluster XIVa). In contrast, smaller particles were associated with higher propionate production.

Overall, the few studies using dietary fiber treatment in PD patients have focused on intestinal constipation $(39,88)$ and its pharmacokinetic effects on drug absorption (88). Metabolites produced in the gut, and composition of gut microbiota in response to dietary fiber treatment, have not been assessed. Cross-sectional studies indicate that the microbial composition in $\mathrm{PD}$ patients present distinct composition from healthy controls (6-10). Although differences in microbial composition varies between $\mathrm{PD}$ and healthy controls across studies, all researchers report decreased abundance of butyrate producers, such as bacteria from Clostridium Cluster XIVa and/or IV (6-10). As butyrate is known to play important physiological roles both within the gastrointestinal tract and in diverse body sites, a dietary fiber approach targeting increases in colonic butyrogenic bacteria (Figure 1) could be beneficial to PD. Studies designed to evaluate dietary fiber effects on bacterial shifts and beneficial metabolite production, especially butyrate, as well as its relation to inflammation, gut permeability, and neurological outcomes in PD, should be conducted. Dietary fibers with specific chemical structures can be selected and/or designed to evaluate if a targeted colonic increase in butyrate and butyrate producers is beneficial to the management of PD outcomes beyond intestinal constipation.

\section{CONCLUSION}

Promoting increases in gut-derived butyrate is a promising approach in PD that could have implications in the management of gut and systemic disturbances. Prebiotic fiber features such as solubility degree, and chemical and physical structures may be important in allowing butyrogenic bacteria to compete against Gram-negative carbohydrate-utilizing bacteria for a more targeted prebiotic approach. The use of specific butyrogenic prebiotic fiber structures in PD models would allow for future pre-clinical studies to understand the effect of gut-produced butyrate in PD.

\section{AUTHOR CONTRIBUTIONS}

TC-J and HR wrote the manuscript. $\mathrm{HR}$ and $\mathrm{BH}$ revised the manuscript.

3. Hawkes CH, Del Tredici K, Braak H. Parkinson's disease: a dual-hit hypothesis. Neuropathol Appl Neurobiol. (2007) 33:599614. doi: 10.1111/j.1365-2990.2007.00874.x

4. Lebouvier T, Chaumette T, Paillusson S, Duyckaerts C, Bruley des Varannes S, Neunlist $\mathrm{M}$, et al. The second brain and Parkinson's disease. Eur J Neurosci. (2009) 30:735-41. doi: 10.1111/j.1460-9568.2009. 06873.x 
5. Forsyth CB, Shannon KM, Kordower JH, Voigt RM, Shaikh M, Jaglin JA, et al. Increased intestinal permeability correlates with sigmoid mucosa alphasynuclein staining and endotoxin exposure markers in early Parkinson's disease. PLoS ONE. (2011) 6:e28032. doi: 10.1371/journal.pone.0028032

6. Keshavarzian A, Green SJ, Engen PA, Voigt RM, Naqib A, Forsyth CB, et al. Colonic bacterial composition in Parkinson's disease. Mov Disord. (2015) 30:1351-60. doi: 10.1002/mds.26307

7. Hill-Burns EM, Debelius JW, Morton JT, Wissemann WT, Lewis MR, Wallen ZD, et al. Parkinson's disease and Parkinson's disease medications have distinct signatures of the gut microbiome. Mov Disord. (2017) 32:73949. doi: $10.1002 / \mathrm{mds} .26942$

8. Petrov VA, Saltykova IV, Zhukova IA, Alifirova VM, Zhukova NG, Dorofeeva YB, et al. Analysis of Gut Microbiota in Patients with Parkinson's Disease. Bull Exp Biol Med. (2017) 162:734-7. doi: 10.1007/s10517-017-3700-7

9. Unger MM, Spiegel J, Dillmann K-U, Grundmann D, Philippeit H, Bürmann J, et al. Short chain fatty acids and gut microbiota differ between patients with Parkinson's disease and age-matched controls. Parkinson Relat Disord. (2016) 32:66-72. doi: 10.1016/j.parkreldis.2016.08.019

10. Li W, Wu X, Hu X, Wang T, Liang S, Duan Y, et al. Structural changes of gut microbiota in Parkinson's disease and its correlation with clinical features. Sci China Life Sci. (2017) 60:1223-33. doi: 10.1007/s11427-016-9001-4

11. Perez-Pardo P, Dodiya HB, Engen PA, Naqib A, Forsyth CB, Green SJ, et al. Gut bacterial composition in a mouse model of Parkinson's disease. Benef Microbes. (2018) 9:799-814. doi: 10.3920/BM2017.0202

12. Gibson GR, Roberfroid MB. Dietary modulation of the human colonic microbiota: introducing the concept of prebiotics. J Nutr. (1995) 125:140112. doi: 10.1093/jn/125.6.1401

13. Gibson GR, Hutkins R, Sanders ME, Prescott SL, Reimer RA, Salminen SJ, et al. Expert consensus document: the International Scientific Association for Probiotics and Prebiotics (ISAPP) consensus statement on the definition and scope of prebiotics. Nat Rev Gastroenterol Hepatol. (2017) 14:491. doi: 10.1038/nrgastro.2017.75

14. Ciorba MA. A gastroenterologist's guide to probiotics. Clin Gastroenterol Hepatol. (2012) 10:960-8. doi: 10.1016/j.cgh.2012.03.024

15. Roberfroid M, Gibson GR, Hoyles L, McCartney AL, Rastall R, Rowland I, et al. Prebiotic effects: metabolic and health benefits. Br J Nutr. (2010) 104:S1-63. doi: 10.1017/S0007114510003363

16. Gibson GR, Fuller R. Aspects of in vitro and in vivo research approaches directed toward identifying probiotics and prebiotics for human use. J Nutr. (2000) 130:391S-5S. doi: 10.1093/jn/130.2.391S

17. Shreiner AB, Kao JY, Young VB. The gut microbiome in health and in disease. Curr Opin Gastroenterol. (2015) 31:69. doi: 10.1097/MOG.0000000000000139

18. Rivière A, Selak M, Lantin D, Leroy F, De Vuyst L. Bifidobacteria and butyrateproducing colon bacteria: importance and strategies for their stimulation in the human gut. Front Microbiol. (2016) 7:979. doi: 10.3389/fmicb.2016.00979

19. Zhang Y-J, Li S, Gan R-Y, Zhou T, Xu D-P, Li H-B. Impacts of gut bacteria on human health and diseases. Int J Mol Sci. (2015) 16:7493. doi: 10.3390/IJMS16047493

20. Hamaker BR, Tuncil YE. A perspective on the complexity of dietary fiber structures and their potential effect on the gut microbiota. J Mol Biol. (2014) 426:3838-50. doi: 10.1016/j.jmb.2014.07.028

21. Codex Alimentarius Commission. Report of the 30th Session of the Codex Committee on Nutrition and Foods for Special Dietary Uses (ALINORM 09/32/26). Rome: Codex Alimentarius Commission (2009).

22. de Jesus Raposo MF, de Morais AMMB, de Morais RMSC. Emergent sources of prebiotics: seaweeds and microalgae. Mar Drugs. (2016) 14:27. doi: $10.3390 / \mathrm{md} 14020027$

23. Ruthes AC, Smiderle FR, Iacomini M. Mushroom heteropolysaccharides: a review on their sources, structure and biological effects. Carbohydr Polym. (2016) 136:358-75. doi: 10.1016/j.carbpol.2015. 08.061

24. Grondin JM, Tamura K, Déjean G, Abbott DW, Brumer H. Polysaccharide utilization loci: fuelling microbial communities. J Bacteriol. (2018) 199:e00860-16. doi: 10.1128/JB.00860-16

25. Rose DJ, Patterson JA, Hamaker BR. Structural differences among alkalisoluble arabinoxylans from maize (Zea mays), rice (Oryza sativa), and wheat (Triticum aestivum) brans influence human fecal fermentation profiles. J Agric Food Chem. (2010) 58:493-9. doi: 10.1021/jf9020416
26. Xu H. Influence of the Structural Complexity of Cereal Arabinoxylans on Human Fecal Fermentation and Their Degradation Mechanism by Gut Bacteria. Theses Diss Available from ProQuest (2012) Available online at: https://docs.lib.purdue.edu/dissertations/AAI3544591 (accessed January 28, 2019).

27. den Besten G, van Eunen K, Groen AK, Venema K, Reijngoud D-J, Bakker $\mathrm{BM}$. The role of short-chain fatty acids in the interplay between diet, gut microbiota, and host energy metabolism. J Lipid Res. (2013) 54:232540. doi: 10.1194/jlr.R036012

28. Holscher HD. Dietary fiber and prebiotics and the gastrointestinal microbiota. Gut Microbes. (2017) 8:172-84. doi: 10.1080/19490976.2017.1290756

29. Zoetendal EG, de Vos WM. Effect of diet on the intestinal microbiota and its activity. Curr Opin Gastroenterol. (2014) 30:189-95. doi: 10.1097/MOG.0000000000000048

30. Vinolo MAR, Rodrigues HG, Nachbar RT, Curi R. Regulation of inflammation by short chain fatty acids. Nutrients. (2011) 3:858-76. doi: 10.3390/nu3100858

31. Tedelind S, Westberg F, Kjerrulf M, Vidal A. Anti-inflammatory properties of the short-chain fatty acids acetate and propionate: a study with relevance to inflammatory bowel disease. World J Gastroenterol. (2007) 13:282632. doi: 10.3748/wjg.v13.i20.2826

32. Liu T, Li J, Liu Y, Xiao N, Suo H, Xie K, et al. Short-chain fatty acids suppress lipopolysaccharide-induced production of nitric oxide and proinflammatory cytokines through inhibition of NF- $\kappa \mathrm{B}$ pathway in RAW264.7 cells. Inflammation. (2012) 35:1676-84. doi: 10.1007/s10753-012-9484-Z

33. Al-Asmakh M, Hedin L. Microbiota and the control of blood-tissue barriers. Tissue Barriers. (2015) 3:1039691. doi: 10.1080/21688370.2015.1039691

34. Hoyles L, Snelling T, Umlai U-K, Nicholson JK, Carding SR, Glen $\mathrm{RC}$, et al. Microbiome-host systems interactions: protective effects of propionate upon the blood-brain barrier. Microbiome. (2018) 6:55. doi: 10.1186/s40168-018-0439-y

35. Oleskin AV, Shenderov BA. Neuromodulatory effects and targets of the SCFAs and gasotransmitters produced by the human symbiotic microbiota. Microb Ecol Heal Dis. (2016) 27:30971. doi: 10.3402/mehd.v27.30971

36. Strandwitz P. Neurotransmitter modulation by the gut microbiota. Brain Res. (2018) 1693:128-33. doi: 10.1016/j.brainres.2018.03.015

37. Grider JR, Piland BE. The peristaltic reflex induced by short-chain fatty acids is mediated by sequential release of 5-HT and neuronal CGRP but not BDNF. Am J Physiol Liver Physiol. (2007) 292:G429-37. doi: 10.1152/ajpgi.00376.2006

38. Winge K, Rasmussen D, Werdelin LM. Constipation in neurological diseases. J Neurol Neurosurg Psychiatry. (2003) 74:13-9. doi: 10.1136/JNNP.74.1.13

39. Barichella M, Pacchetti C, Bolliri C, Cassani E, Iorio L, Pusani C, et al. Probiotics and prebiotic fiber for constipation associated with Parkinson disease. Neurology. (2016) 87:1274-80. doi: 10.1212/WNL.0000000000003127

40. St. Laurent R, O’Brien LM, Ahmad ST. Sodium butyrate improves locomotor impairment and early mortality in a rotenone-induced Drosophila model of Parkinson's disease. Neuroscience. (2013) 246:382-90. doi: 10.1016/j.neuroscience.2013.04.037

41. Mony L, Kew JN, Gunthorpe MJ, Paoletti P. Allosteric modulators of NR2B-containing NMDA receptors: molecular mechanisms and therapeutic potential. Br J Pharmacol. (2009) 157:130117. doi: 10.1111/j.1476-5381.2009.00304.x

42. Paiva I, Pinho R, Pavlou MA, Hennion M, Wales P, Schütz A-L, et al. Sodium butyrate rescues dopaminergic cells from alpha-synuclein-induced transcriptional deregulation and DNA damage. Hum Mol Genet. (2017) 26:2231-46. doi: 10.1093/hmg/ddx114

43. Zhou W, Bercury K, Cummiskey J, Luong N, Lebin J, Freed CR. Phenylbutyrate up-regulates the DJ-1 protein and protects neurons in cell culture and in animal models of Parkinson disease. J Biol Chem. (2011) 286:14941-51. doi: 10.1074/jbc.M110.211029

44. Kidd SK, Schneider JS. Protection of dopaminergic cells from MPP+mediated toxicity by histone deacetylase inhibition. Brain Res. (2010) 1354:172-8. doi: 10.1016/j.brainres.2010.07.041

45. Salama AF, Ibrahim W, Tousson E, Sakr S, Masoud A, Akela MA, et al. Epigenetic study of Parkinson's disease in experimental animal model. Int $J$ Clin Exp Neurol. (2015) 3:11-20. doi: 10.12691/IJCEN-3-1-3

46. Roediger WEW. Utilization of nutrients by isolated epithelial cells of the rat colon. Gastroenterology. (1982) 83:4249. doi: 10.1016/S0016-5085(82)80339-9 
47. Husted AS, Trauelsen M, Rudenko O, Hjorth SA, Schwartz TW. GPCR-mediated signaling of metabolites. Cell Metab. (2017) 25:77796. doi: 10.1016/J.CMET.2017.03.008

48. Kim MH, Kang SG, Park JH, Yanagisawa M, Kim CH. Short-chain fatty acids activate GPR41 and GPR43 on intestinal epithelial cells to promote inflammatory responses in mice. Gastroenterology. (2013) 145:396406.e10. doi: 10.1053/j.gastro.2013.04.056

49. D’Souza WN, Douangpanya J, Mu S, Jaeckel P, Zhang M, Maxwell JR, et al. Differing roles for short chain fatty acids and GPR43 agonism in the regulation of intestinal barrier function and immune responses. PLoS ONE. (2017) 12:e0180190. doi: 10.1371/journal.pone.0180190

50. Christiansen CB, Gabe MBN, Svendsen B, Dragsted LO, Rosenkilde MM, Holst JJ. The impact of short-chain fatty acids on GLP-1 and PYY secretion from the isolated perfused rat colon. Am J Physiol Liver Physiol. (2018) 315:G53-65. doi: 10.1152/ajpgi.00346.2017

51. Tolhurst G, Heffron H, Lam YS, Parker HE, Habib AM, Diakogiannaki E, et al. Short-chain fatty acids stimulate glucagon-like peptide-1 secretion via the G-protein-coupled receptor FFAR2. Diabetes. (2012) 61:36471. doi: $10.2337 / \mathrm{db} 11-1019$

52. Liu J, Wang F, Liu S, Du J, Hu X, Xiong J, et al. Sodium butyrate exerts protective effect against Parkinson's disease in mice via stimulation of glucagon like peptide-1. J Neurol Sci. (2017) 381:176-81. doi: 10.1016/j.jns.2017.08.3235

53. Santos AL, Lindner AB. Protein posttranslational modifications: roles in aging and age-related disease. Oxid Med Cell Longev. (2017) 2017:119. doi: 10.1155/2017/5716409

54. Davie JR. Inhibition of histone deacetylase activity by butyrate. J Nutr. (2003) 133:2485S-93S. doi: 10.1093/jn/133.7.2485S

55. Chang PV, Hao L, Offermanns S, Medzhitov R. The microbial metabolite butyrate regulates intestinal macrophage function via histone deacetylase inhibition. Proc Natl Acad Sci USA. (2014) 111:2247-52. doi: 10.1073/pnas.1322269111

56. Egorin MJ, Yuan Z-M, Sentz DL, Plaisance K, Eiseman JL. Plasma pharmacokinetics of butyrate after intravenous administration of sodium butyrate or oral administration of tributyrin or sodium butyrate to mice and rats. Cancer Chemother Pharmacol. (1999) 43:445-53. doi: 10.1007/s002800050922

57. Knudsen KEB, Serena A, Canibe N, Juntunen KS. New insight into butyrate metabolism. Proc Nutr Soc. (2003) 62:81-6. doi: 10.1079/PNS20 02212

58. Robertson MD, Bickerton AS, Dennis AL, Vidal H, Frayn KN. Insulinsensitizing effects of dietary resistant starch and effects on skeletal muscle and adipose tissue metabolism. Am J Clin Nutr. (2005) 82:55967. doi: 10.1093/ajen.82.3.559

59. Priebe MG, Wang H, Weening D, Schepers M, Preston T, Vonk RJ. Factors related to colonic fermentation of nondigestible carbohydrates of a previous evening meal increase tissue glucose uptake and moderate glucose-associated inflammation. Am J Clin Nutr. (2010) 91:90-7. doi: 10.3945/ajcn.2009. 28521

60. Wolever TM, Chiasson JL. Acarbose raises serum butyrate in human subjects with impaired glucose tolerance. Br J Nutr. (2000) 84:57-61. doi: 10.1017/S0007114500001239

61. Ktsoyan ZA, Mkrtchyan MS, Zakharyan MK, Mnatsakanyan AA, Arakelova KA, Gevorgyan ZU, et al. Systemic concentrations of short chain fatty acids are elevated in salmonellosis and exacerbation of familial mediterranean fever. Front Microbiol. (2016) 7:776. doi: 10.3389/fmicb.2016. 00776

62. Sampson TR, Debelius JW, Thron T, Janssen S, Shastri GG, Ilhan ZE, et al. Gut microbiota regulate motor deficits and neuroinflammation in a model of Parkinson's disease. Cell. (2016) 167:1469-80.e12. doi: 10.1016/j.cell.2016.11.018

63. Mulak A. A controversy on the role of short-chain fatty acids in the pathogenesis of Parkinson's disease. Mov Disord. (2018) 33:398401. doi: $10.1002 / \mathrm{mds} .27304$

64. Kratsman N, Getselter D, Elliott E. Sodium butyrate attenuates social behavior deficits and modifies the transcription of inhibitory/excitatory genes in the frontal cortex of an autism model. Neuropharmacology. (2016) 102:13645. doi: 10.1016/j.neuropharm.2015.11.003
65. Vital M, Karch A, Pieper DH. Colonic butyrate-producing communities in humans: an overview using omics data. mSystems. (2017) 2:e0013017. doi: 10.1128/MSYSTEMS.00130-17

66. Louis P, Flint HJ. Diversity, metabolism and microbial ecology of butyrateproducing bacteria from the human large intestine. FEMS Microbiol Lett. (2009) 294:1-8. doi: 10.1111/j.1574-6968.2009.01514.x

67. Walker AW, Duncan SH, Louis P, Flint HJ. Phylogeny, culturing, and metagenomics of the human gut microbiota. Trends Microbiol. (2014) 22:26774. doi: 10.1016/J.TIM.2014.03.001

68. El Aidy S, Van den Abbeele P, Van de Wiele T, Louis P, Kleerebezem M. Intestinal colonization: how key microbial players become established in this dynamic process. BioEssays. (2013) 35:913-23. doi: 10.1002/bies.2013 00073

69. Van den Abbeele P, Belzer C, Goossens M, Kleerebezem M, De Vos WM, Thas O, et al. Butyrate-producing Clostridium cluster XIVa species specifically colonize mucins in an in vitro gut model. ISME J. (2013) 7:94961. doi: 10.1038/ismej.2012.158

70. Lopetuso LR, Scaldaferri F, Petito V, Gasbarrini A. Commensal Clostridia: leading players in the maintenance of gut homeostasis. Gut Pathog. (2013) 5:23. doi: 10.1186/1757-4749-5-23

71. Walker AW, Duncan SH, McWilliam Leitch EC, Child MW, Flint HJ. pH and peptide supply can radically alter bacterial populations and short-chain fatty acid ratios within microbial communities from the human colon. Appl Environ Microbiol. (2005) 71:3692-700. doi: 10.1128/AEM.71.7.3692-3700.2005

72. Belenguer A, Duncan SH, Holtrop G, Anderson SE, Lobley GE, Flint HJ. Impact of $\mathrm{pH}$ on lactate formation and utilization by human fecal microbial communities. Appl Environ Microbiol. (2007) 73:652633. doi: 10.1128/AEM.00508-07

73. Duncan SH, Louis P, Thomson JM, Flint HJ. The role of $\mathrm{pH}$ in determining the species composition of the human colonic microbiota. Environ Microbiol. (2009) 11:2112-22. doi: 10.1111/j.1462-2920.2009.01931.x

74. Walker AW, Duncan SH, Harmsen HJM, Holtrop G, Welling GW, Flint HJ. The species composition of the human intestinal microbiota differs between particle-associated and liquid phase communities. Environ Microbiol. (2008) 10:3275-83. doi: 10.1111/j.1462-2920.2008.01717.x

75. Pereira FC, Berry D. Microbial nutrient niches in the gut. Environ Microbiol. (2017) 19:1366-78. doi: 10.1111/1462-2920.13659

76. Duncan SH, Belenguer A, Holtrop G, Johnstone AM, Flint HJ, Lobley GE. Reduced dietary intake of carbohydrates by obese subjects results in decreased concentrations of butyrate and butyrate-producing bacteria in feces. Appl Environ Microbiol. (2007) 73:1073-8. doi: 10.1128/AEM.02340-06

77. Sheridan PO, Martin JC, Lawley TD, Browne HP, Harris HMB, BernalierDonadille A, et al. Polysaccharide utilization loci and nutritional specialization in a dominant group of butyrate-producing human colonic Firmicutes. Microb Genomics. (2016) 2:e000043. doi: 10.1099/mgen.0.000043

78. Leitch ECM, Walker AW, Duncan SH, Holtrop G, Flint HJ. Selective colonization of insoluble substrates by human faecal bacteria. Environ Microbiol. (2007) 9:667-79. doi: 10.1111/j.1462-2920.2006.01186.x

79. Mirande C, Kadlecikova E, Matulova M, Capek P, Bernalier-Donadille A, Forano E, et al. Dietary fibre degradation and fermentation by two xylanolytic bacteria Bacteroides xylanisolvens $\mathrm{XB1A^{ \textrm {T } }}$ and Roseburia intestinalis XB6B4 from the human intestine. J Appl Microbiol. (2010) 109:451-60. doi: 10.1111/j.1365-2672.2010.04671.x

80. Neyrinck AM, Possemiers S, Verstraete W, De Backer F, Cani PD, Delzenne NM. Dietary modulation of clostridial cluster XIVa gut bacteria (Roseburia spp.) by chitin-glucan fiber improves host metabolic alterations induced by high-fat diet in mice. J Nutr Biochem. (2012) 23:519. doi: 10.1016/j.jnutbio.2010.10.008

81. Cantu-Jungles TM, Ruthes AC, El-Hindawy M, Moreno RB, Zhang X, Cordeiro LMC, et al. In vitro fermentation of Cookeina speciosa glucans stimulates the growth of the butyrogenic Clostridium cluster XIVa in a targeted way. Carbohydr Polym. (2018) 183:219-29. doi: 10.1016/j.carbpol.2017. 12.020

82. Martínez I, Lattimer JM, Hubach KL, Case JA, Yang J, Weber CG, et al. Gut microbiome composition is linked to whole grain-induced immunological improvements. ISME J. (2013) 7:269-80. doi: 10.1038/ismej. 2012.104 
83. Salonen A, Lahti L, Salojärvi J, Holtrop G, Korpela K, Duncan SH, et al. Impact of diet and individual variation on intestinal microbiota composition and fermentation products in obese men. ISME J. (2014) 8:221830. doi: 10.1038/ismej.2014.63

84. La Rosa SL, Kachrimanidou V, Buffetto F, Pope PB, Pudlo NA, Martens EC, et al. Wood-Derived Dietary Fibers Promote Beneficial Human Gut Microbiota. mSphere. (2019) 4:18. doi: 10.1128/mSphere.00554-18

85. Duncan SH, Russell WR, Quartieri A, Rossi M, Parkhill J, Walker AW, et al. Wheat bran promotes enrichment within the human colonic microbiota of butyrate-producing bacteria that release ferulic acid. Environ Microbiol. (2016) 18:2214-25. doi: 10.1111/1462-2920.13158

86. Tuncil YE, Thakkar RD, Marcia ADR, Hamaker BR, Lindemann SR. Divergent short-chain fatty acid production and succession of colonic microbiota arise in fermentation of variously-sized wheat bran fractions. Sci Rep. (2018) 8:16655. doi: 10.1038/s41598-018-34912-8

87. Baxter NT, Schmidt AW, Venkataraman A, Kim KS, Waldron C, Schmidt TM. Dynamics of human gut microbiota and short-chain fatty acids in response to dietary interventions with three fermentable fibers. mBio. (2019) 10:e2566-18. doi: 10.1128/MBIO.02566-18

88. Astarloa R, Mena MA, Sánchez V, de la Vega L, de Yébenes JG. Clinical and pharmacokinetic effects of a diet rich in insoluble fiber on Parkinson disease. Clin Neuropharmacol. (1992) 15:375-80.

Conflict of Interest Statement: The authors declare that the research was conducted in the absence of any commercial or financial relationships that could be construed as a potential conflict of interest.

Copyright (c) 2019 Cantu-Jungles, Rasmussen and Hamaker. This is an open-access article distributed under the terms of the Creative Commons Attribution License (CC BY). The use, distribution or reproduction in other forums is permitted, provided the original author(s) and the copyright owner(s) are credited and that the original publication in this journal is cited, in accordance with accepted academic practice. No use, distribution or reproduction is permitted which does not comply with these terms. 